Phys Med Biol 2015, 60 (7): 2047-2073

\title{
A rigid motion correction method for helical computed tomography (CT)
}

\author{
J-H Kim ${ }^{1}$, J Nuyts ${ }^{2}$, A Kyme ${ }^{1,3}$, Z Kuncic ${ }^{4}$ and \\ R Fulton ${ }^{1,3,4,5}$ \\ ${ }^{1}$ Discipline of Medical Imaging and Sciences, University of Sydney, NSW 2006, \\ Australia \\ ${ }^{2} \mathrm{KU}$ Leuven - University of Leuven, Department of Imaging and Pathology, \\ Nuclear Medicine \& Molecular imaging, Medical Imaging Research Center \\ (MIRC), B-3000, Leuven, Belgium \\ ${ }^{3}$ Brain and Mind Research Institute, University of Sydney, NSW 2050, Australia \\ ${ }^{4}$ School of Physics, University of Sydney, NSW 2050, Australia \\ ${ }^{5}$ Department of Medical Physics, Westmead Hospital, Westmead, NSW 2145, \\ Australia \\ E-mail: roger.fulton@sydney.edu.au
}

\begin{abstract}
We propose a method to compensate for six degree-of-freedom rigid motion in helical $\mathrm{CT}$ of the head. The method is demonstrated in simulations and in helical scans performed on a 16-slice CT scanner. Scans of a Hoffman brain phantom were acquired while an optical motion tracking system recorded the motion of the bed and the phantom. Motion correction was performed by restoring projection consistency using data from the motion tracking system, and reconstructing with an iterative fully 3D algorithm. Motion correction accuracy was evaluated by comparing reconstructed images with a stationary reference scan. We also investigated the effects on accuracy of tracker sampling rate, measurement jitter, interpolation of tracker measurements, and the synchronization of motion data and CT projections. After optimization of these aspects, motion corrected images corresponded remarkably closely to images of the stationary phantom with correlation and similarity coefficients both above 0.9. We performed a simulation study using volunteer head motion and found similarly that our method is capable of compensating effectively for realistic human head movements. To the best of our knowledge, this is the first practical demonstration of generalized rigid motion correction in helical CT. Its clinical value, which we have yet to explore, may be significant. For example it could reduce the necessity for repeat scans and resource-intensive anesthetic and sedation procedures in patient groups prone to motion, such as young children. It is not only applicable to dedicated CT imaging, but also to hybrid PET/CT and SPECT/CT, where it could also ensure an accurate CT image for lesion localization and attenuation correction of the functional image data.
\end{abstract}

Submitted to: Phys. Med. Biol.

Keywords: Motion estimation, motion compensation, computed tomography, image reconstruction, reconstruction algorithms. 


\section{Introduction}

One of the major sources of image artifacts in computed tomography (CT) is patient motion, which creates inconsistencies between acquired projections, leading to distortion and blurring when images are reconstructed (Popilock et al., 2008, Yazdi and Beaulieu, 2008, Schöndube et al., 2013). These motion artifacts may lead to false diagnosis, or in extreme cases, render images uninterpretable (Edlow et al., 2000). Head motion is a common problem in young patients who are often sedated or anesthetized to prevent motion (Kaste, 2004, Wachtel et al., 2009). According to the latest available data, over 70 million CT scans are performed annually in the USA alone, of which approximately $10 \%$ are performed in children (Brenner, 2010). Moreover, a recent survey of CT practice in developing countries revealed that about $75 \%$ of pediatric CT scans were of the head (Vassileva et al., 2012). Due to the relatively high radiation dose associated with CT scanning, it is undesirable to repeat the scan if motion occurs, particularly in children who have a much higher estimated lifetime risk of radiation-induced cancer than adults (Brenner et al., 2001, Brenner and Hall, 2007). In adults, head motion is a problem for patients suffering from claustrophobia or a mental or behavioral incapacity, and in patients with head trauma (Lee et al., 2005). In a recent study (Fahmi et al., 2013), head movements classified as moderate or severe were observed in $25 \%$ of 103 patients with acute ischemic stroke during CT brain perfusion scans.

Whereas methods for the correction of head motion using external motion monitoring devices have been published for single photon emission tomography (SPECT), e.g. Fulton et al. (1994), Weisenberger et al. (2005), and positron emission tomography (PET), e.g. Picard and Thompson (1997), Fulton et al. (2002), Herzog et al. (2005), Bloomfield et al. (2003), no equivalent method has been demonstrated for helical CT imaging. Such a method would not only be of value in standalone CT imaging, but also in the hybrid imaging modalities PET/CT and SPECT/CT where an accurate CT image is essential for correct anatomical localization and attenuation correction of the functional data.

To reduce the likelihood of motion artifacts, CT manufacturers have made scanners faster by increasing the number of detector rows and the rate of rotation of the x-ray source and detector. This is not a complete solution, however, since there is still a possibility of patient motion during the scan. The effect of such motion is illustrated in Fig. 1, in a patient with suspected head trauma. The study was performed with a state-of-the-art helical CT scanner with a rotation time of $0.35 \mathrm{~s}$ and 80 detector rows. Motion of this kind occurs frequently in clinical practice, and can only be remedied by repeating the scan (Fahmi et al., 2013, Al-Shakhrah and Al-Obaidi, 2003).

Other ways to reduce the likelihood of patient motion include general anesthesia, sedation (Kaste, 2004, Wachtel et al., 2009) and the use of restraining devices for head and neck imaging (Beyer et al., 2005). Breath-holding is used to reduce respiratory motion artifacts (Katsuda et al., 2005) while gating techniques are effective in mitigating the effects of periodic (e.g. cardiac and respiratory) motions (Ritchie et al., 1994, Desjardins et al., 2004). For patients prone to uncontrolled head motion, such 


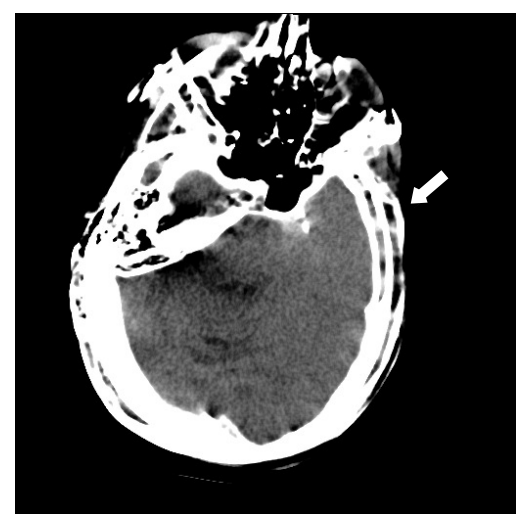

Figure 1. A slice from a helical CT scan showing head motion artifacts in a 27 year old male patient with suspected brain trauma. The arrow indicates an area of ghosting which causes the skull to appear in 3 different locations. Other areas of ghosting can be seen in the image. The scan was performed on a Toshiba Aquilion Prime CT scanner $(120 \mathrm{kVp}, 200 \mathrm{~mA}$, pitch 0.625 , slice thickness $4 \mathrm{~mm})$. (Image courtesy of Westmead Hospital, Sydney, Australia).

as infants, anesthesia and sedation are effective, but demanding of time and resources, and anesthesia in particular introduces the added risks of hypoxemia, respiratory depression and oxygen desaturation, which can result in long-term consequences (Malviya et al., 2000).

Another way to mitigate motion-induced artifacts is to apply retrospective motion correction. To date several methods have been proposed for correcting for rigid motion (Wang and Vannier, 1995, Yu and Wang, 2007, Zafar, 2011, Bodensteiner et al., 2007, Jacobson et al., 2008, Ens et al., 2010, Bhowmik, et al., 2012, Schäfer et al., 2004) and nonrigid motion (Schäfer et al., 2004, Crawford et al., 1996, Ritchie et al., 1996, Roux et al., 2004, Desbat et al., 2007, Taguchi and Kudo, 2008, Lu and Mackie, 2002, Marchant et al., 2011, Isola et al., 2008, Rit et al., 2009, Schretter et al., 2009) in various types of $\mathrm{CT}$ imaging. In this paper we address the correction of rigid motion, characterized by six degrees-of-freedom (d.o.f.) (3 rotations and 3 translations), in helical CT. Of the rigid motion correction methods mentioned above, most have been intended for cone beam CT (CBCT) (Bodensteiner et al., 2007, Jacobson et al., 2008, Ens et al., 2010, Bhowmik, et al., 2012, Schäfer et al., 2004) and relatively few methods have been proposed for fan beam (Yu and Wang, 2007) or helical CT (Wang and Vannier, 1995, Zafar, 2011). Motion correction is arguably simpler in CBCT since the entire object will normally be in the field of view at all times, whereas in helical $\mathrm{CT}$ the object is always truncated. This truncation can complicate the application of analytical motion correction algorithms to helical CT, and may explain why published analytical methods (Wang and Vannier, 1995, Yu and Wang, 2007, Zafar, 2011) appear to be limited to in-plane motion, and to have only been investigated in simulations.

Virtually all suggested approaches to motion correction in CT require information about the time course of the object motion during the scan. Some studies have obtained rigid motion data using an external tracking system, (Westermann and 
Hauser, 2000, Wagner et al., 2003) or by analysis of the acquired CT projection data in CBCT (Wang and Vannier, 1995, Yu and Wang, 2007, Bodensteiner et al., 2007, Jacobson et al., 2008, Ens et al., 2010, Bhowmik, et al., 2012, Schäfer et al., 2004, Linney et al., 2001), or by following the motion of anatomical landmarks or radio-opaque markers in the image or projection domain (Bodensteiner et al., 2007, Jacobson et al., 2008, Ens et al., 2010, Bhowmik, et al., 2012, Schäfer et al., 2004). Noo et al. (2001) and Mennessier et al. (2009) used markers for geometrical calibration of $\mathrm{CBCT}$, which is equivalent to estimating a rigid motion of the object. Landmarks and markers have also been used to deduce nonrigid motion (Ritchie et al., 1996, Lu and Mackie, 2002, Marchant et al., 2011, Isola et al., 2008). However, deducing rigid motion from helical CT projections appears to be an unsolved problem, possibly due to the limited time that identifiable landmarks remain in the field of view as the bed moves axially.

We recently demonstrated the feasibility of tracking fast six d.o.f. rigid motion during helical CT scans using an optical motion tracking system (Kim et al., 2013). In the present work we describe a method of compensating for large and rapid six d.o.f. rigid motion during helical $\mathrm{CT}$ using this tracking technique. This method relies on accurate and frequent motion estimates during the scan, and a fully 3D iterative reconstruction algorithm. Here, feasibility is demonstrated in CT scans of a brain phantom performed on a clinical CT scanner, and in simulations of realistic human motion obtained from a volunteer. To the best of our knowledge, this is the first practical demonstration of rigid motion correction in helical CT.

\section{Methods}

\subsection{Motion Correction Principle}

In $\mathrm{CT}$, projection views are acquired sequentially as the source and detector rotate about the imaged object. Rigid object motion during acquisition introduces inconsistencies between successive projection views. Reconstructing with a conventional algorithm, ignoring the motion, results in artifacts. However if the motion is rigid, projection consistency may be restored by translating and rotating the $\mathrm{CT}$ detector and source at each projection view by the inverse of the object motion, to create a virtual scanner trajectory. The projection data within each view remain unchanged. The rigid motion of the imaged object may be obtained in various ways, e.g. using an optical motion tracking system, calibrated to provide measurements directly in a CT coordinate system with origin at the scanner isocentre (Kim et al., 2013).

During reconstruction the (back)projection is computed using the virtual scanner trajectory (Fig. 2). Theoretically, by reconstructing from consistent projections, we eliminate motion effects, including zoom which will change if the imaged object moves towards or away from the detector. This effect is computed accurately during forward and backprojection because we accurately model the motion during reconstruction. However, it is important to note that motion during the scan creates a motiondependent scanning trajectory that cannot be guaranteed to be sufficient for exact 


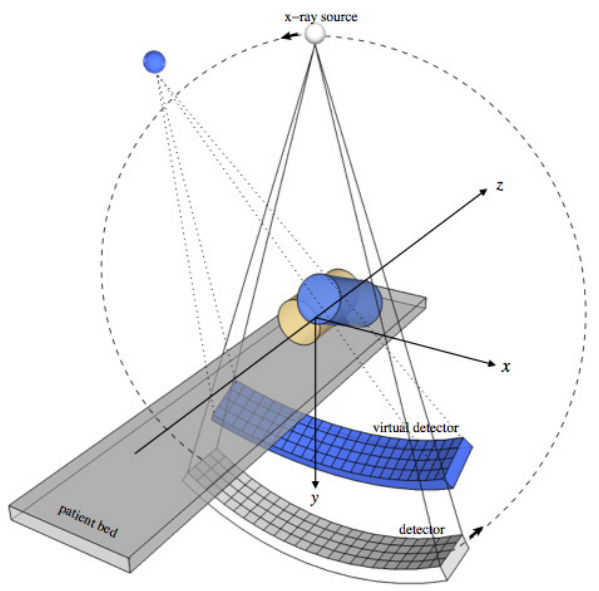

Figure 2. The motion correction scheme: object (yellow cylinder) moves to a new pose (blue), projection consistency can be restored by translating and rotating the CT detector and source (grey) at each projection view by the inverse of the object's motion from its initial pose to obtain a new, virtual source/detector pose (blue). Attributing the acquired data to the virtual pose makes it consistent with a scenario in which the object remained stationary.

reconstruction. For example, in the extreme case, rotation of the object in the same direction and at the same speed as the motion of the x-ray source would result in projection data at only one angle, which clearly would be insufficient for reconstruction.

\subsection{Motion-corrected reconstruction}

Reconstructions were performed with a fully 3D implementation of the maximum likelihood transmission reconstruction (MLTR) algorithm (Nuyts et al., 1998). MLTR maximizes the logarithm of the likelihood $L(\mu)$, given by

$$
L(\mu)=\sum_{i} y_{i} \ln \hat{y}_{i}-\hat{y}_{i}-\ln \left(y_{i} !\right)
$$

where $y_{i}$ is the measured intensity along projection line $i, \hat{y}_{i}$ is the estimate of $y_{i}$ at the current reconstruction, and $y_{i}$ is assumed to be a Poisson realization of $\hat{y}_{i}$. Ignoring scatter, detector blurring and the polychromatic energy spectrum, the estimate $\hat{y}_{i}$ can be written as

$$
\hat{y}_{i}=b_{i} \exp \left(-\sum_{j} l_{i j} \mu_{j}\right)
$$

where $b_{i}$ is the number of photons detected in the absence of absorber (blank scan), $l_{i j}$ is the effective intersection length of projection line $i$ with voxel $j$, and $\mu_{j}$ is the linear attenuation coefficient at voxel $j$. An update step for maximizing Eq. (1) is

$$
\Delta \mu_{j}=-\frac{\partial L}{\partial \mu_{j}} / \sum_{k} \frac{\partial^{2} L}{\partial \mu_{j} \partial \mu_{k}}
$$


A derivation is given in Van Slambrouck et al. (2012). Inserting Eq. (1) in (3) gives the MLTR algorithm

$$
\mu_{j}^{\text {new }}=\mu_{j}+\frac{\sum_{i} l_{i j}\left(\hat{y}_{i}-y_{i}\right)}{\sum_{i}\left(l_{i j} \hat{y}_{i} \sum_{k} l_{i k}\right)}
$$

Since the index $i$ runs over all projection lines, it combines the view number and the detector pixel. Correction for patient motion is achieved by modifying the trajectory of the $\mathrm{CT}$ source and detector, i.e. by changing the values $l_{i j}$ such that they represent projections using the modified trajectory.

The projector/backprojector was implemented using both the ray-tracing (Joseph, 1982) and distance-driven (De Man and Basu, 2004) approaches. Except where otherwise indicated, we used ray-tracing as this method was less computationally demanding in our implementation. Our implementation of MLTR enabled motion correction by allowing the pose of each projection to be individually specified in a scanner coordinate system. When motion was included, the detector and source were (conceptually) translated and rotated, and the (back)projection computed using this moved CT-system.

\subsection{Real CT scans (Hoffman brain phantom)}

The motion correction method was tested in real CT scans of a moving brain phantom. All experiments were performed on a Siemens Biograph 16 PET/CT scanner (Siemens Medical Solutions USA, Inc., Knoxville, TN), which incorporates a standard Siemens Somatom Sensation 16-slice helical CT scanner. The phantom, a 3D Hoffman brain phantom (Hoffman et al., 1990), is usually filled with radioactive water for use in SPECT and PET, but in our CT imaging experiments it contained air. This gave the phantom a complex attenuation profile, due to multiple regions of zero attenuation coefficient surrounding perspex (soft tissue) structures. Its sharp edges and sudden changes of attenuation coefficient were expected to increase the sensitivity of calculated metrics to any distortion or misalignment of the motion corrected image relative to the reference image.

2.3.1. Motion Tracking Setup An optical motion tracking system (Polaris Spectra, Northern Digital Inc., Waterloo, Canada) was placed at the rear of the scanner on a tripod (Fig. 3(a)). It reported the six d.o.f. pose of a rigid-body target consisting of four retro-reflective disks $(0.6 \mathrm{~cm}$ radius) attached to the underside of the $3 \mathrm{D}$ Hoffman brain phantom as the three rotations, $R_{x}(\psi), R_{y}(\theta)$ and $R_{z}(\phi)$, and three translations, $T_{x}, T_{y}$ and $T_{z}$. Specifying the order of rotations as $R_{x}(\psi)$, followed by $R_{y}(\theta)$ and lastly $R_{z}(\phi)$, the pose, $P$, was represented as the $4 \times 4$ transformation matrix

$$
\begin{aligned}
& P= \\
& {\left[\begin{array}{cccc}
\cos \phi \cos \theta & \cos \phi \sin \theta \sin \psi-\sin \phi \cos \psi & \cos \phi \sin \theta \cos \psi+\sin \phi \sin \psi & T_{x} \\
\sin \phi \cos \theta & \sin \phi \sin \theta \sin \psi+\cos \phi \cos \psi & \sin \phi \sin \theta \cos \psi-\cos \phi \sin \psi & T_{y} \\
-\sin \theta & \cos \theta \sin \psi & \cos \theta \cos \psi & T_{z} \\
0 & 0 & 0 & 1
\end{array}\right] .}
\end{aligned}
$$


An additional target comprising three disks was attached to the front edge of the bed so that bed motion could also be recorded. The tracker was operated in passive mode to record the poses of the phantom and bed simultaneously at $60 \mathrm{~Hz}$. The Polaris system has been used extensively in PET motion correction (Beyer et al., 2005, Fulton et al., 2002, Bloomfield et al., 2003). A review of its performance can be found in Elfring et al. (2010).

A cross-calibration procedure (Kim et al., 2013) was performed to determine the $4 \times 4$ transformation matrix relating the tracker and CT scanner coordinate systems. This enabled the pose data to be converted to the CT coordinate space centred at the isocentre of the scanner (Fig. 2) for use by the motion correction algorithm.

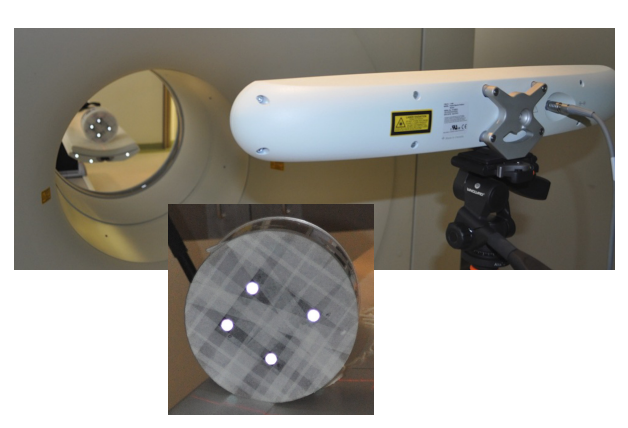

(a) Setup for motion tracking

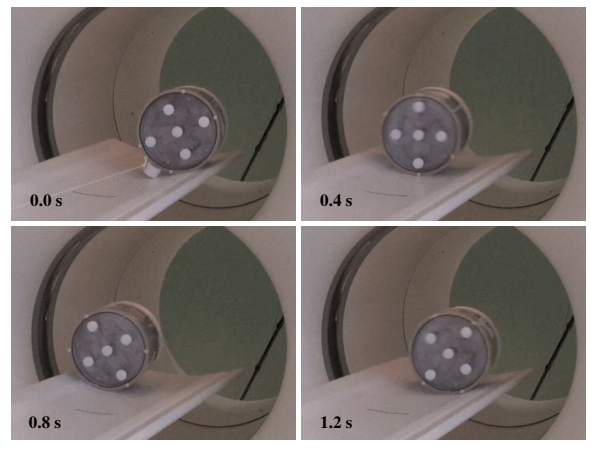

(b) Phantom motion

Figure 3. (a) Motion tracking setup on the Siemens Biograph 16 PET/CT scanner. The CT scanner port is visible at the far end of the bore. The phantom with four reflective markers attached can be seen on the bed. The inset gives a closer view. Three reflective markers can also be seen on the bed; (b) Selected video frames from a $1.2 \mathrm{~s}$ portion of the moving phantom scan showing the rate and oscillatory nature of the motion. Removal of the wedge occurs at top left. Elapsed times shown are relative to this frame. One leg of the tripod supporting the motion tracking system can be seen in the background.

2.3.2. CT Scans In CT experiments, the phantom was initially supported by a wedge in an elevated position on the curved surface of the bed. A stationary helical CT scan of the phantom was performed to serve as a reference. Then without moving the phantom, a repeat CT scan was started. During this scan the wedge was removed by pulling a string from outside the room. This caused the phantom to roll from side to side over the concave bed (see Fig. 3(b)). The oscillatory motion continued with diminishing amplitude for the remainder of the scan. The peak velocity in the $x$ (horizontal) direction of a reference point on the rear surface of the phantom was calculated from motion tracking data as $\approx 180 \mathrm{~mm} \mathrm{~s}^{-1}$. A video camera captured the experiment from the bed side of the gantry, while the optical motion tracking system positioned behind the gantry tracked the motion of the phantom and bed.

With the exception of one set of scans performed at a higher pitch of 1.0, all CT scans used the scanner's built-in protocol for routine pediatric head imaging with the scan and reconstruction parameters shown in Table 1. These scans contained 47,287 
views. To accelerate reconstruction, projections were rebinned by summing groups of four consecutive projections.

In the scan performed with a pitch of 1.0, motion was applied in the same way, but was not identical as the method of applying motion was not reproducible. Other differences were in the initial pose of the phantom, the axial scan length $(285 \mathrm{~mm})$, the table feed per rotation $(13 \mathrm{~mm})$ and the total scan time $(10.52 \mathrm{~s})$. Tube voltage, tube current, rotation time, and collimation were unchanged. The acquired scans contained 24,445 views. Projections were rebinned in the same way as previously and images were reconstructed with the same voxel size, number of iterations and subsets.

Table 1. CT scanning and reconstruction parameters.

\begin{tabular}{ll}
\hline Parameter & Value \\
\hline Detector rows & 16 \\
Tube voltage $(\mathrm{kVp})$ & 120 \\
Tube current $(\mathrm{mAs})$ & 150 \\
Rotation time (s) & 0.5 \\
Projections per rotation & 1160 \\
Rebinned projections per rotation & 290 \\
Flying focal spot & Off \\
Collimation (mm) & $16 \times 0.75$ \\
Table feed per rotation (mm) & 6.5 \\
Pitch & 0.5 \\
Axial coverage (mm) & 276 \\
Scan duration (s) & 20.36 \\
Rebinned projections & 11821 \\
Rebinned projection interval $(\mathrm{ms})$ & 1.724 \\
Reconstructed voxel dimensions $(\mathrm{mm})$ & $1.0 \times 1.0 \times 1.0$ \\
\hline
\end{tabular}

2.3.3. Motion Data Processing The reconstruction software accounts for the motion of the bed and the rotation of the CT source and detector. This is implemented as a helical trajectory around a stationary object. Therefore, to deal with additional motion of the object, we need the motion relative to a coordinate system that is fixed to the bed, with the origin in the centre of the scanned volume. However, the motions are measured in a coordinate system in which the bed and therefore also the scanned volume are moving. At the mid-scan time, the centre of the scanned volume coincides perfectly with the centre of the CT, whereas for all other time points, a correction for the change in bed position is required. Therefore, for every phantom pose measurement represented in homogeneous coordinates (Paul, 1982) by the $4 \times 4$ matrix $P_{i}$, we needed to compute the 'difference' between the pose of the bed, $B_{i}$, at the corresponding time and the bed pose at the mid-scan time, $B_{m}$. The resulting pose of the phantom with bed motion removed, $P_{i}^{\prime}$, was then given by

$$
P_{i}^{\prime}=B_{m} B_{i}^{-1} P_{i}
$$

Then the motion of the phantom at each measurement time, relative to its pose at the start of the scan $\left(P_{r e f}^{\prime}\right)$, was calculated as

$$
M_{i}=P_{i}^{\prime} P_{r e f}^{\prime-1} \text {. }
$$


This ensured that the motion correction procedure produced a reconstruction in the same pose as the reference scan. However, in practice, the reference pose could be any desired pose. $P_{r e f}^{\prime}$ was calculated as the mean of the last 30 pose measurements before the motion scan commenced. For calculation of the mean pose, $x, y$ and $z$ positions were averaged arithmetically, while cosine averaging (Stavdahl et al., 2005) was applied to rotations.

To synchronize motion estimates $M_{i}$ with corresponding CT projections to a first approximation, the motion tracker measurement corresponding to the start of the CT scan was identified by detection of bed motion in the $z$ direction. Thereafter, corresponding motion tracker measurements and CT projections were identified by finding the closest match between bed $z$ positions reported by the tracking system and corresponding information stored in the file header for each projection view. We refer to this method as nearest-neighbour synchronization.

2.3.4. Motion Correction Reference scans and motion scans without motion correction were reconstructed using MLTR without use of motion information. In MLTR with motion correction, the synchronized motion transformations (Sec. 2.3.3) were inverted and applied to the detector and source, creating a virtual scanner trajectory where the (back)projection was computed as described in Sec. 2.2. The motion correction method assumes that everything in the scanner field of view moves rigidly according to the motion applied, which is not true for the patient bed. Therefore the bed was removed from the data before reconstruction by subtracting a forward projection of an image of the bed from the log-converted sinogram.

2.3.5. Quantitative Evaluation of Motion Correction Accuracy The accuracy of motion corrected transaxial images was assessed by comparison with corresponding images from the stationary reference scan. To reduce the influence of the static background on similarity measures, metric calculations were confined to voxels within an ROI enclosing the phantom. Three quantitative similarity metrics were evaluated:

(i) RMSE: The square root of the mean of the squared differences of pixel intensities of two $2 \mathrm{D}$ images $f$ and $g$ calculated as

$$
R M S E=\sqrt{\frac{1}{N} \sum_{i=1}^{N}\left[f_{i}-g_{i}\right]^{2}}
$$

where $N$ is the number of pixels.

(ii) CC: The Pearson correlation coefficient which measures the linear dependence between two images as

$$
C C=\frac{\sum_{i=1}^{N}\left(f_{i}-\bar{f}\right)\left(g_{i}-\bar{g}\right)}{\sqrt{\sum_{i=1}^{N}\left(f_{i}-\bar{f}\right)^{2}} \sqrt{\sum_{i=1}^{N}\left(g_{i}-\bar{g}\right)^{2}}}
$$

where $\bar{f}$ and $\bar{g}$ are the mean pixel values of the two images. 
(iii) MSSIM: The mean structural similarity index (Wang et al., 2004), a measure of the similarity of two images in terms of luminance $l(f, g)$, contrast $c(f, g)$, and structure $s(f, g)$ that is designed to provide a good approximation of perceptual image quality, is given by

$$
M S S I M=\frac{1}{N} \sum_{i=1}^{N}\left\{[l(f, g)]^{\alpha}[c(f, g)]^{\beta}[s(f, g)]^{\gamma}\right\}_{i}
$$

where $\alpha, \beta, \gamma$ are weighting factors (set to 1 in this study) that adjust the relative importance of the 3 components. Here the luminance comparison is defined by Wang et al as

$$
l(f, g)=\frac{2 \mu_{f} \mu_{g}+\left(K_{1} L\right)^{2}}{\mu_{f}^{2}+\mu_{g}^{2}+\left(K_{1} L\right)^{2}}
$$

where $\mu$ is the mean pixel intensity in the image, $K_{1}$ is a small constant $\ll 1$, and $L$ is the dynamic range of the pixel values (255 in this study). Contrast comparison employs the function

$$
c(f, g)=\frac{2 \sigma_{f} \sigma_{g}+\left(K_{2} L\right)^{2}}{\sigma_{f}^{2}+\sigma_{g}^{2}+\left(K_{2} L\right)^{2}}
$$

where $\sigma$ is the standard deviation of the pixel values in the image, and $K_{2} \ll 1$. Finally, the structure comparison is based on the function

$$
s(f, g)=\frac{\sigma_{f g}+\frac{1}{2}\left(K_{2} L\right)^{2}}{\sigma_{f} \sigma_{g}+\frac{1}{2}\left(K_{2} L\right)^{2}}
$$

where $\sigma_{f g}$ is given by

$$
\sigma_{f g}=\frac{1}{N-1} \sum_{i=1}^{N}\left(f_{i}-\mu_{f}\right)\left(g_{i}-\mu_{g}\right) .
$$

Following Wang et al we set $K_{1}$ and $K_{2}$ to 0.01 and 0.03 , respectively.

RMSE and CC were calculated from images with pixel values in Hounsfield Units (HUs), whereas for MSSIM, images were linearly scaled to have pixel values ranging from 0 to 255. Thus, from Eqns. (8)-(10), RMSE was calculated in HU while CC and MSSIM were dimensionless. Metric evaluations were performed on 150 transaxial slices (1 mm thick) covering the majority of the phantom. The means $(\overline{\mathrm{RMSE}}, \overline{\mathrm{CC}}$, and

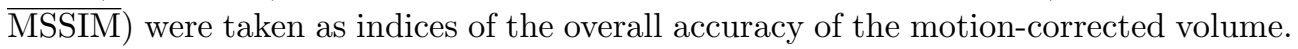
Standard deviations were also computed to indicate the slice-to-slice variation in the calculated metrics.

\subsection{Optimization}

We refer to the motion correction method so far described as the 'default' motion correction method. After an initial assessment of the accuracy of this method, we investigated the sensitivity of the motion correction accuracy to variations in the method. 
2.4.1. Tracker sampling rate The relationship between tracker sampling rate and motion correction accuracy was investigated by down-sampling the tracker data obtained at $60 \mathrm{~Hz}$ to simulate sampling rates of 48,40,30,25, 20, 15, 10 and $5 \mathrm{~Hz}$. Intermediate pose measurements were neglected, which resulted in the same spatial transformation being applied at an increasing number of consecutive projections as the sample rate was reduced. Motion corrected images in a common reference pose $\left(P_{r e f}^{\prime}\right)$ were reconstructed using each subsampled motion data set, and image metrics were calculated.

2.4.2. Motion data filtering and interpolation A Savitzky-Golay (SG) filter (Savitzky and Golay, 1964) was employed with the aim of reducing jitter in the pose measurements, while preserving the underlying signal. The SG filter fits a polynomial of the form $y=a_{0}+a_{1} x+a_{2} x^{2}+\ldots+a_{n} x^{n}$ to the data points by least squares. A set of filter coefficients was pre-calculated for the given number of data points and degree of the polynomial and convolved with the data for each d.o.f. to produce the fitted curve. In our experiments we empirically determined that satisfactory jitter suppression could be achieved using a 17-point kernel and a polynomial of degree $n=2$. We compared motion-corrected reconstructions obtained with and without application of the filter to the motion tracker data. In the same way we also tested the effect of linear interpolation between successive motion samples (both filtered and unfiltered) to enable a specific, interpolated motion transformation to be applied at every CT projection. This interpolation was applied independently in each d.o.f.

2.4.3. Synchronization As an alternative to synchronization based on nearestneighbour comparison of bed positions (Sec. 2.3.3), and after identifying the motion tracker measurement corresponding to the first CT projection in the same way, timestamps were assigned to motion samples and rebinned projections assuming that they were acquired at constant rates throughout the scan $(1 / 60 \mathrm{~s}$ and $1 / 580 \mathrm{~s}$ for tracker and projection data, respectively). For the CT projections we also compared these time stamps with timing information stored in the CT file header for each projection, and found negligible differences of $\approx 3.5 \mu$ s on average. SG filtering was applied, and linear interpolation as described above was used to assign individual motion transformations at all CT projection views. Due to the uncertainty in precisely identifying the tracker measurement corresponding to the start of the CT scan, the optimal synchronization was determined by applying a range of time shifts in units of $1 \mathrm{CT}$ projection interval to all tracker timestamps, repeating motion correction with each time shift, and identifying the shift that optimized the (dis)similarity metrics described in Sec. 2.3.5. The optimal time shift was taken as the average of the shifts identified by the three metrics.

\subsection{Assessment of Number of Views}

As mentioned above the motion-corrected scanner trajectory may, for some motion patterns, provide insufficient sampling for exact reconstruction. As a first attempt to detect possible sampling insufficiency, we backprojected a unit projection for every 
view using the distance-driven backprojector, and counted for each voxel the number of views contributing to its backprojection value. While this is not a robust measure of sufficiency, we hypothesised that sampling is more likely to be insufficient at locations in the image where the backprojection value is low.

\subsection{Simulation Study with Volunteer Motion and Anthropomorphic Head Phantom}

2.6.1. CT simulations Although a phantom study could reveal the feasibility of correcting for rigid motion, the pattern and magnitude of its motion was considered unlikely to resemble that of a human head. Therefore, we also assessed the efficacy of motion correction in a simulated Siemens Somatom Sensation 16-slice CT scan with realistic human head motion obtained from a volunteer lying in a CT scanner. The simulation was of a voxelized 3D anthropomorphic head phantom (PBU-60, Kyoto Kagaku Co. Ltd, Kyoto, Japan) with a voxel size of $1.5 \mathrm{~mm} \times 1.5 \mathrm{~mm} \times 1.5 \mathrm{~mm}$, created by scanning the phantom on a 128 -slice Siemens Definition AS CT scanner. Simulated scan parameters were the same as used in the phantom scans (Table 1).

The realistic head motion data were acquired with a volunteer (a healthy 25 year old male) lying supine on the scanning bed of a Siemens Biograph mCT PET/CT scanner (Siemens Medical Solutions USA, Inc., Knoxville, TN) using an Optitrack (NaturalPoint Inc, Corvallis, OR USA) optical motion tracking system which comprised three Flex 13 infrared cameras positioned at the rear of the scanner (see Fig. 4(a)). This system allowed faster sampling (up to $120 \mathrm{~Hz}$ ) and easier marker attachment than the tracking system used in the phantom scans. A cross-calibration procedure was carried out prior to motion tracking as described in Sec. 2.3.1, to determine the $4 \times 4$ transformation matrix relating the tracker and CT isocentre coordinate system.

The volunteer wore a beanie with four retro-reflective velcro markers (spheres $14 \mathrm{~mm}$ in diameter) attached as shown in Fig. 4(b). Compared with the Polaris system which requires all markers to be co-planar and in a predetermined arrangement, the Optitrack is much more flexible. Markers are tracked as a rigid point cloud, and can be tracked on curved surfaces. The desired number of markers can be placed wherever desired on the head. The volunteer's head was placed on a headrest in the CT fieldof-view (FOV) with the centre of the head at the isocentre of the scanner using the laser guide. The volunteer was asked to move his head freely for $15 \mathrm{~s}$. The pose of the volunteer's head and the patient bed were simultaneously recorded at $120 \mathrm{~Hz}$.

The first $3 \mathrm{~s}$ of the volunteer motion data were used in the simulations. The raw tracker data were firstly converted to the $\mathrm{CT}$ isocentre coordinate system. To reduce jitter in the resulting data, represented as a series of $4 \times 4$ matrices $\{P\}$, a 17-point second degree SG polynomial (Sec. 2.4.2) was fitted to the data for each d.o.f. yielding the smooth pose data, $\{S\}$. For the simulations at pitch $0.5,\{S\}$ was repeated three times. As simulations at pitch 1.5 were 3 times faster ( $3 \mathrm{~s}$ duration), a single use of $\{S\}$ was sufficient. Motion relative to the reference pose of the head was calculated (as in Eq. 7) using

$$
M_{i}=S_{i} S_{r e f}^{-1}
$$




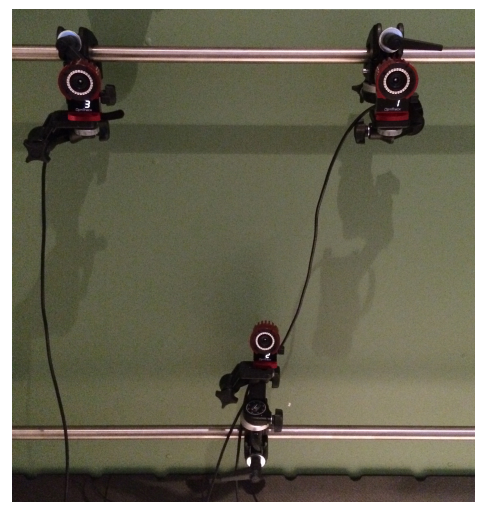

(a) Optitrack motion tracking system

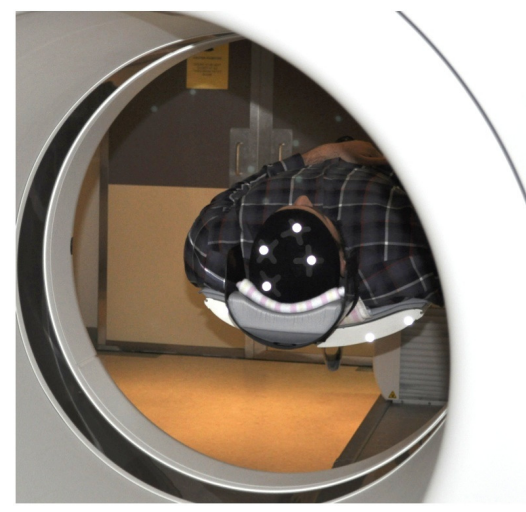

(b) Volunteer with head markers positioned for motion tracking

Figure 4. Volunteer motion tracking setup. (a) Three infrared cameras mounted on the wall behind the scanner. (b) The volunteer positioned in the CT scanner field of view.

where $S_{i}, S_{\text {ref }}$ and $M_{i}$ were $4 \times 4$ matrices representing the pose at measurement time $i$, the reference pose, and the calculated motion, respectively. The first pose of the volunteer was used as the reference pose. The motion data, $\{M\}$, were then linearly interpolated to match the timestamps of each simulated projection angle using the same method as described in Sec. 2.4.3. To simulate motion, the phantom was rotated and shifted by applying the appropriate motion transformation at each projection angle, and the CT-projections were computed using the conventional scanning trajectory, using distance-driven projection, assuming a monochromatic beam and no scatter. This was adequate for our purpose as our sole aim was to test the ability of the motion correction method to cope with a scanning trajectory resulting from real human head motion. It was not our objective to study more subtle effects such as beam hardening.

2.6.2. Motion correction Simulations of the stationary phantom were reconstructed without motion correction using the standard MLTR algorithm, while simulations with motion were reconstructed using the same algorithm, with and without motion correction (Sec. 2.2). The distance-driven (back)projector was used in all simulations. During reconstruction, in contrast to the simulation method, the object was treated as stationary, while the CT-projections were computed after rotating and shifting the CT-gantry. This introduced some mismatch between simulation and reconstruction, because the different approaches led to different interpolation coefficients when the projections were computed. Motion-corrected reconstruction of each CT scan with simulated motion was performed using two different types of motion information; motion calculated from the same ('true') pose data $\{S\}$ that had been used to create the motion simulation, and motion calculated from 'realistic' pose data, $\{R\}$, obtained by adding jitter in the form of simulated Gaussian noise to $\{S\}$. In the latter we made the magnitude of the jitter as realistic as possible by scaling the noise in each d.o.f. to 
the root mean square of differences between $\{S\}$ and $\{P\}$ for that d.o.f. after applying Eq. 6.

Motion transformations, $\{Q\}$, were then calculated from $\{R\}$ w.r.t. the same reference pose, $S_{r e f}$, as in Eq. 15. Finally, the motion transformations to be applied at each projection angle during reconstruction were obtained by fitting the polynomial function described in Sec. 2.4.2 to $\{Q\}$ in each d.o.f., and interpolating to the number of $\mathrm{CT}$ projections based on timestamps.

Motion correction with 'true' pose data was intended to show the effect of motion correction when the motion was known exactly. Motion correction with 'realistic' pose data was intended to show whether motion correction could be effective when the motion data were affected by realistic measurement jitter. Three metrics were calculated on simulated images using the same methods as described in Sec. 2.3.5 for quantitative comparison.

\section{Results}

\subsection{Phantom study}

3.1.1. Motion Tracking Fig. 5 shows the six d.o.f. motion of the phantom during the study shown in Fig. 3(b), in CT isocentre coordinates, relative to its pose at the start of the scan. It illustrates the oscillatory nature of the motion, which involved motion in all six d.o.f., including rotations of at least $10^{\circ}$ about all axes, and translations of up to $103 \mathrm{~mm}$. This motion was much larger than that typically expected in patients, based on data obtained by Wagner et al. in 20 patients during helical CT scans, who reported rotations of up to $2.3^{\circ}$ and translations of up to $5 \mathrm{~mm}$ (Wagner et al., 2003). However we hypothesized that motion of the magnitude tested here could be encountered in some patients. For example, Fahmi et al. (2013) reported rotations and translations exceeding $60^{\circ}$ and $60 \mathrm{~mm}$, respectively, during helical CT perfusion scans in patients with acute ischemic stroke.

3.1.2. Motion Correction Fig. 6 shows reconstructed slices from the same scan, reconstructed with and without (default) motion correction, and the corresponding slices from the reference scan in which the phantom was stationary. In this, and all subsequent images shown, the slices depicted are, from left to right, transaxial, coronal, and sagittal. The slices without motion correction, Fig. 6(a), exhibited severe motion artifacts. The motion corrected image, Fig. 6(c), on the other hand, was clearly similar to the reference, Fig. 6(b), with motion artifacts substantially eliminated. Some irregularities were visible at the edges of the phantom and moiré patterns appeared in uniform regions, which may be attributed to interference between the rays and the pixels. We later observed that this effect was reduced when using the distance-driven (back)projector (Fig. 12(c)).

Table 2 shows image similarity metric values with and without default motion correction for the images shown in Fig. 6, using the reference study as the gold standard. The mean and standard deviation of each metric were calculated over 150 reconstructed slices. All the metrics showed marked improvement when motion 


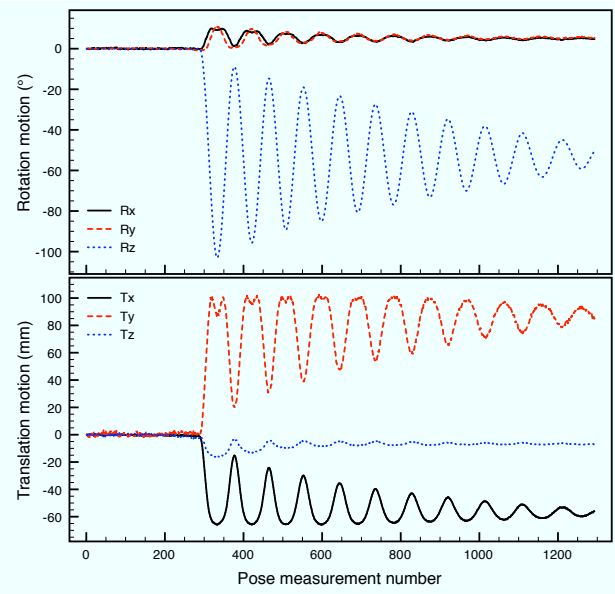

Figure 5. Rotations (top) and translations (bottom) of the phantom motion during the scan shown in Fig. 3(b).

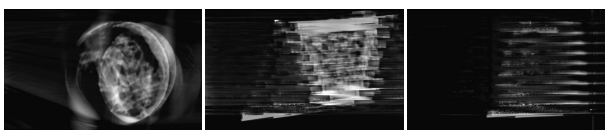

(a) Motion - Uncorrected

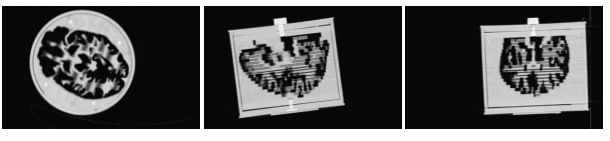

(b) Reference - No motion

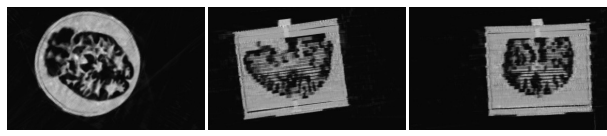

(c) Motion corrected - default

Figure 6. Transaxial coronal and sagittal reconstructed slices from the moving phantom study shown in Fig. 3(b). (a) Moving phantom, no motion correction. (b) Stationary phantom. (c) Moving phantom, with motion correction. Pose data were acquired at $60 \mathrm{~Hz}$. The parallel lines visible in the coronal and sagittal slices of the reference and motion corrected scans are due to slabs of acrylic material from which the phantom is constructed. [Window level, $W L=-200 \mathrm{HU}$, window width, $W W=+2000 \mathrm{HU}]$.

correction was applied. In calculating these metrics we assumed that the motion of the bed was perfectly reproducible, and that there was no motion of the phantom between the reference and motion scans.

Table 2. Reconstruction accuracy with and without default motion correction.

\begin{tabular}{llll}
\hline & $\overline{\mathrm{RMSE}}$ & $\overline{\mathrm{CC}}$ & $\overline{\mathrm{MSSIM}}$ \\
\hline Uncorrected & $721.6(83.0)$ & $0.054(0.185)$ & $0.377(0.068)$ \\
Corrected & $176.9(38.1)$ & $0.912(0.031)$ & $0.748(0.053)$ \\
\hline
\end{tabular}

Values are mean (s.d.) of 150 transaxial slices. 


\subsection{Optimization}

3.2.1. Effect of Tracker Sampling Rate Motion corrected images obtained with the default motion correction method and different tracker sampling rates are presented in Fig. 7. Qualitatively similar motion corrected reconstructions were obtained with sampling rates of $20 \mathrm{~Hz}, 30 \mathrm{~Hz}$ and $48 \mathrm{~Hz}$, while artifacts were clearly visible at $10 \mathrm{~Hz}$. In a similar analysis for continuous motion in PET, a sampling rate of $20 \mathrm{~Hz}$ was found to be sufficient for awake rats (Kyme et al., 2011).

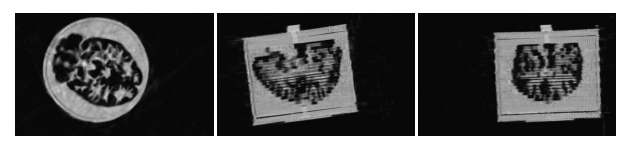

(a) $48 \mathrm{~Hz}$

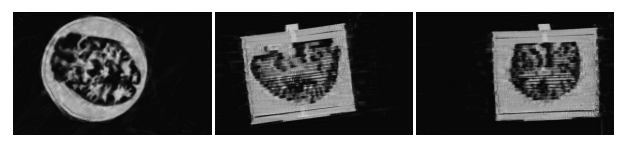

(c) $20 \mathrm{~Hz}$

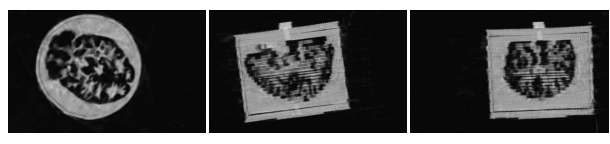

(b) $30 \mathrm{~Hz}$

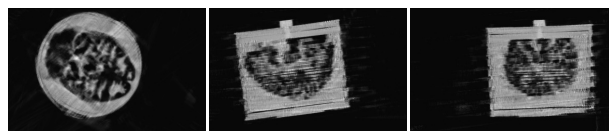

(d) $10 \mathrm{~Hz}$

Figure 7. Motion corrected images (default method) for different tracker sampling rates. Artifacts are clearly visible at the edges of the phantom at sampling rates below $30 \mathrm{~Hz}$. [ $W L=-200 \mathrm{HU}, W W=+2000 \mathrm{HU}]$.

The quantitative relationship between tracker sampling rate and reconstruction accuracy is shown in Fig. 8. All metrics showed that accuracy was greatest at the highest tracker sampling rate available $(60 \mathrm{~Hz})$. Below $30 \mathrm{~Hz}$ there was a gradual decline in motion correction accuracy.

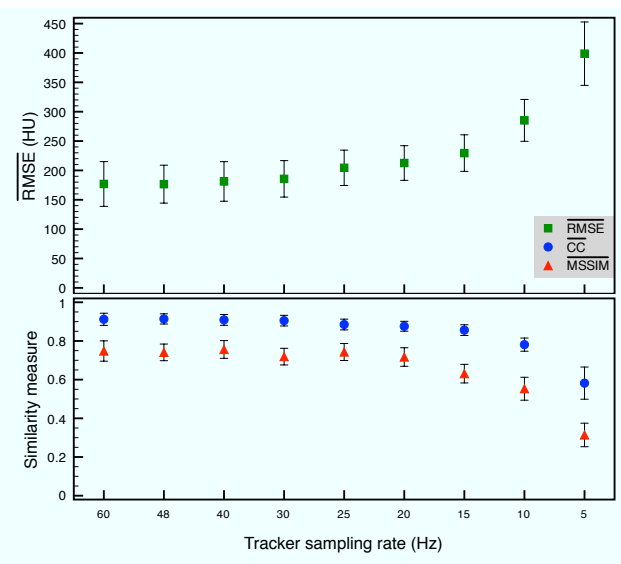

Figure 8. The relationship between quantitative accuracy of the default motion corrected image and tracker sampling rate by three different metrics. Data represent the mean ( \pm 1 s.d.) over 150 transaxial slices.

3.2.2. Effect of motion data filtering and interpolation The effects of smoothing and linearly interpolating raw $60 \mathrm{~Hz}$ tracker data over a typical $345 \mathrm{~ms}$ segment of the 
moving phantom scan are presented in Fig. 9. The solid black line corresponds to data used in the default motion correction method with nearest-neighbour synchronization (Sec. 2.3.3) and no interpolation between tracker samples. Simple linear interpolation between sample points produced the curve composed of green crosses. Interpolation enabled a unique transformation to be applied to each CT projection, but did not eliminate jitter. Combining linear interpolation and the SG filter (short red dashes) gave a much smoother result. Metrics of motion correction accuracy evaluated with five different treatments of the raw motion data in the moving phantom study are shown in Fig. 10. All three metrics agreed well. The lowest accuracy was obtained with the default motion correction method which used nearest-neighbour synchronization. Introducing linear interpolation without filtering improved accuracy, but on its own it was not as effective as SG filtering. Combining SG filtering with interpolation gave further improvement.
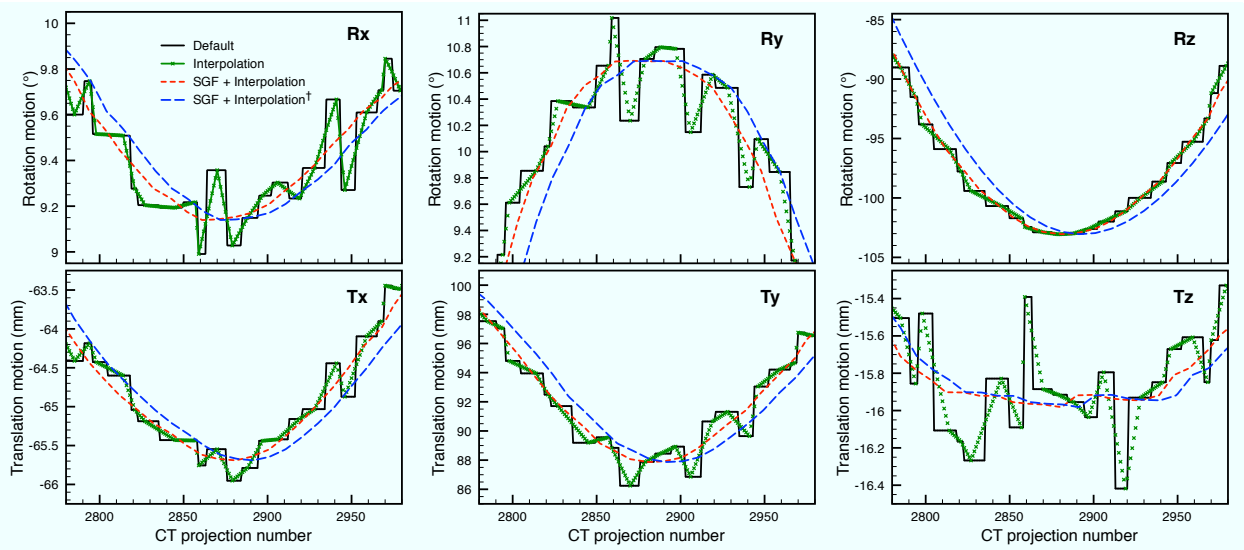

Figure 9. Various combinations of interpolation and filtering applied to raw six d.o.f. motion data. Without filtering or interpolation (solid line) measurements are affected by jitter, and the same motion transformation must be applied to all $\mathrm{CT}$ projections between tracker samples. Interpolation alone (green crosses) fails to remove measurement jitter. A smoother result is obtained with the SG filter and interpolation (red dashes). The light blue dashed curve is also with SG filter and linear interpolation, but synchronized assuming constant tracker and $\mathrm{CT}$ projection acquisition rates and zero time shift.

3.2.3. Synchronization Fig. 10 also shows that the highest accuracy for the optimal $60 \mathrm{~Hz}$ sampling rate was obtained by combining SG filtering, linear interpolation and the timestamp-based synchronization described in Sec. 2.4.3 to obtain the motion data represented by the blue dashes in Fig. 9, and then applying an optimized time-shift to these data. Fig. 11 demonstrates the variation in metric values versus time shift. The optimal time shift was found to be $13 \mathrm{CT}$ projection intervals $(\approx 22.4 \mathrm{~ms})$. This yielded the motion-corrected images shown in Fig. 12(b). Metric values corresponding to these five different treatments of the motion data are shown in the first five rows of Table 3. We observed that the three metrics were always consistent in identifying the 


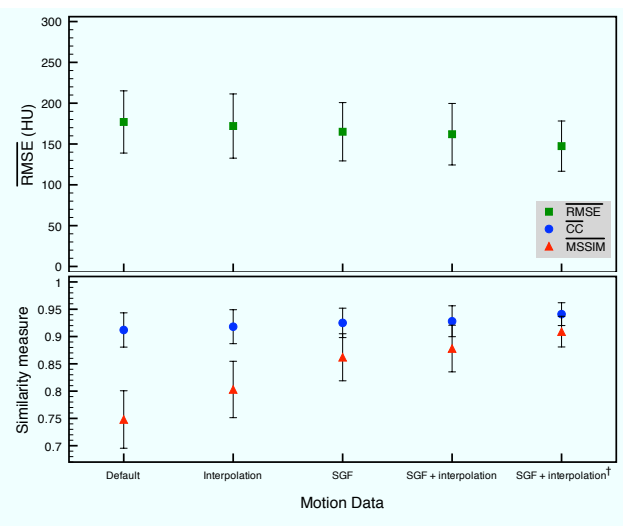

Figure 10. Motion correction accuracy quantified by three image metrics, with various combinations of filtering method and interpolation applied to the raw motion data. Data represent the mean ( \pm 1 s.d.) over 150 transaxial slices. ${ }^{\dagger}$ indicates timestamp-based synchronization with optimal time shift.

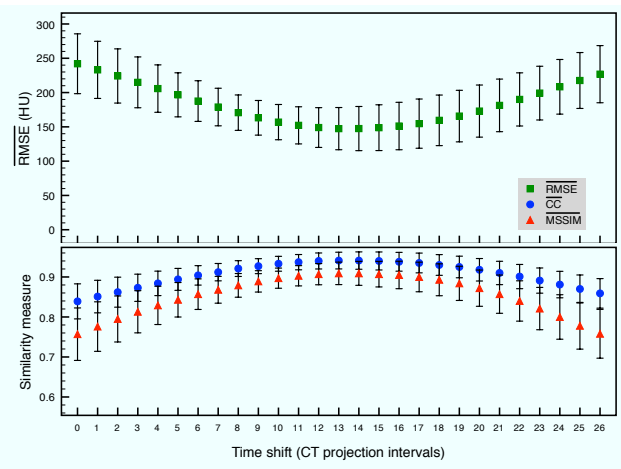

Figure 11. Motion correction accuracy vs. applied time-shift. Data represent the mean ( \pm 1 s.d.) over 150 transaxial slices.

optimal time shift, and that we could have obtained the same result using any one of them instead of averaging their results.

3.2.4. Comparison with distance-driven (back)projection Fig. 12(c) shows the result of substituting distance-driven (back)projection for the ray-tracing method used in Fig. 12(b). Distance-driven (back)projection improved motion correction accuracy for all metrics (lower row of Table 3) and reduced moiré patterns in the reconstructed images. However, motion-corrected reconstruction was approximately 3.5 times slower when the distance-driven (back)projector was used.

\subsection{Sensitivity to Pitch}

Fig. 13 shows results of motion correction in a phantom study with similar motion, performed with a pitch of 1.0. Motion correction accuracy metrics for this study 


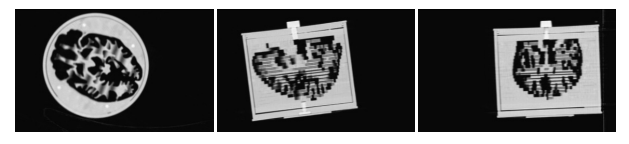

(a) Reference (No motion)

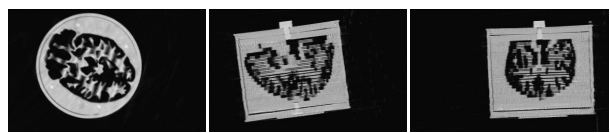

(b) Optimized correction (Ray-tracing)

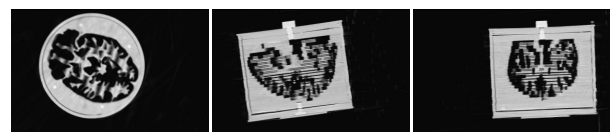

(c) Optimized correction (Distance-driven)

Figure 12. Reconstructed image slices. Optimized motion correction was obtained with $60 \mathrm{~Hz}$ sampling, timestamp-based synchronization, SG filtering, linear interpolation, and optimized time-shift. Motion corrected images are shown for both ray-tracing and distance-driven (back)projection methods. The reference images are reproduced from Fig. 6(b) for ease of comparison. [WL $=-200 \mathrm{HU}$, $W W=+2000 \mathrm{HU}]$

Table 3. Reconstruction accuracy with motion data filtering, interpolation and different synchronization methods

\begin{tabular}{|c|c|c|c|}
\hline & $\overline{\mathrm{RMSE}}$ & $\overline{\mathrm{CC}}$ & $\overline{\text { MSSIM }}$ \\
\hline Default & $176.9(38.1)$ & $0.912(0.031)$ & $0.748(0.053)$ \\
\hline Interpolation & $171.7(39.3)$ & $0.918(0.031)$ & $0.803(0.052)$ \\
\hline SGF & $165.3(35.7)$ & $0.925(0.027)$ & $0.862(0.043)$ \\
\hline SGF + interpolation & $161.5(37.7)$ & $0.928(0.028)$ & $0.878(0.043)$ \\
\hline $\mathrm{SGF}+$ interpolation $^{\dagger}$ & $147.4(30.8)$ & $0.942(0.022)$ & $0.909(0.030)$ \\
\hline $\mathrm{SGF}+$ interpolation ${ }^{\dagger \ddagger}$ & $136.9(30.4)$ & $0.949(0.019)$ & $0.925(0.027)$ \\
\hline
\end{tabular}

Values are mean (s.d.) of 150 transaxial slices.

†Timestamp-based synchronization method with optimal time shift.

${ }^{\ddagger}$ Distance-driven (back)projector.

are shown in Table 4. Motion correction, after processing the motion data with SG filtering, linear interpolation, and an optimized time shift (7 CT projection intervals) substantially restored the shape of the phantom and reduced motion artifacts. However, some motion artifacts remained. Results of assessing data sufficiency in this study as described in Sec. 2.5 are shown in Fig. 14 for pitch values of (a) 0.5 and (b) 1.0. Images in the upper two rows were windowed to highlight areas with backprojection values lower than a threshold value, which we arbitrarily set to 0.6 times the mean backprojection value of all voxels in the $3 \mathrm{D}$ volume. Voxels exceeding this threshold are displayed in white. Reconstructed images exhibiting artifacts in all 3 planes are shown in the lower row with a window $[W L=-200 \mathrm{HU}$, $W W=+2000 \mathrm{HU}]$. There were no regions with prominent reduced sampling at lower pitch. For pitch of 1.0, cursors overlaid on the images indicate corresponding locations in 3D. We observed that artifacts did indeed occur in regions seen by few views, suggesting that the artifacts were due to sampling insufficiency. This suggests that certain combinations of object motion and pitch can result in undersampling artifacts in reconstructed images, and that for a given type of motion, increasing the pitch 
increases the potential for artifacts due to data insufficiency. It was not possible to perform a systematic evaluation of the effect of pitch alone on motion correction accuracy, due to the effect of a change in pitch on other scanning parameters such as scan duration and the timing of motion relative to CT source-detector motion.

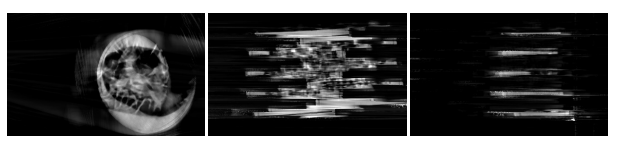

(a) No Correction

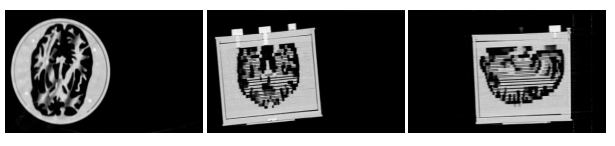

(b) Reference

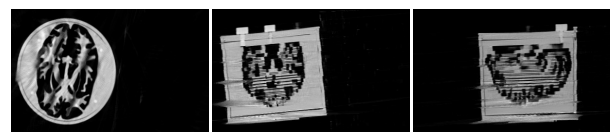

(c) Optimized

Figure 13. Motion correction in a $\mathrm{CT}$ scan performed with a pitch of 1.0. $[\mathrm{WL}=-200 \mathrm{HU}, \mathrm{WW}=+2000 \mathrm{HU}]$.

Table 4. Motion correction accuracy in a study with pitch $=1.0$.

\begin{tabular}{lccc}
\hline & $\overline{\mathrm{RMSE}}$ & $\overline{\mathrm{CC}}$ & $\overline{\mathrm{MSSIM}}$ \\
\hline Uncorrected & $752.8(100.37)$ & $0.035(0.161)$ & $0.384(0.107)$ \\
Corrected & $262.6(42.38)$ & $0.800(0.046)$ & $0.813(0.055)$ \\
\hline
\end{tabular}

Values are mean (s.d.) of 150 transaxial slices.

\subsection{Simulation study}

Fig. 15 shows the motion of the volunteer that was used in the simulations. There was motion in all six d.o.f., of up to $4.6^{\circ}$ and $7 \mathrm{~mm}$ for rotations and translations, respectively.

Slice and projection images from the voxelized phantom are shown in Fig. 16(a). Results of CT scans simulated with pitch factors of 0.5 and 1.5 are shown in Figs. 16(b) and 16(c), respectively. For both pitch values, the images reconstructed without motion correction (top row) were severely distorted by the motion. The motion-corrected images obtained from simulations of the true motion (third row) corresponded very closely to those of the stationary simulation and the original phantom. When motion correction was based on pose data affected by jitter (lower row), some perceptible artifacts remained that were visibly worse at pitch of 1.5 than at 0.5 .

These results showed that accurate motion correction of realistic human head motion could be achieved if the true motion was precisely known. Some streaks were observed near bony structures in the motion-corrected images at both pitch values in the same location as in the corresponding reference images. An assessment of the effect of the number of views (Sec 2.5) revealed relatively high backprojection values in these areas. Rather than data insufficiency, the streaking was attributed to mismatch in 


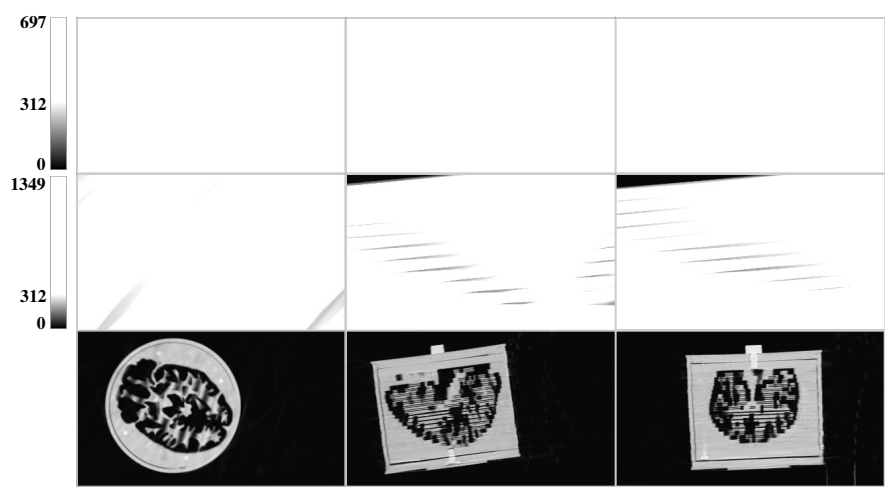

(a) Pitch $=0.5$

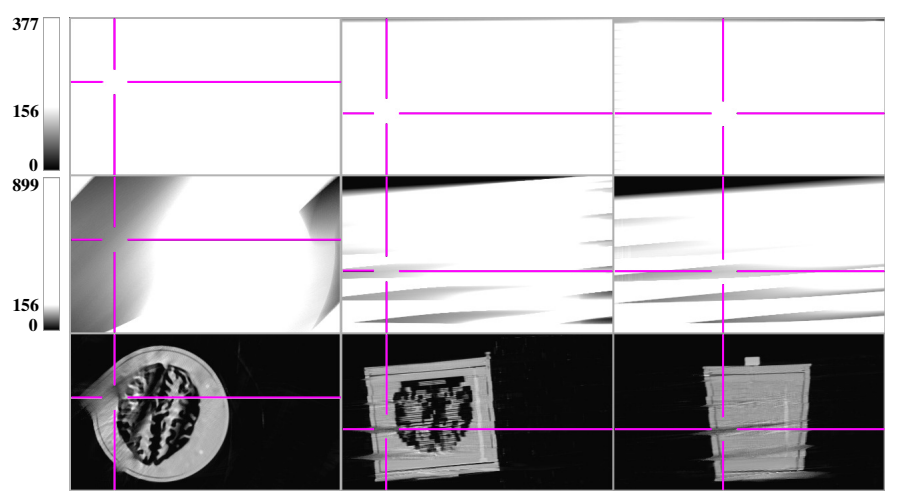

(b) Pitch $=1.0$

Figure 14. Backprojection of a uniform sinogram for the experiments with (a) pitch of 0.5, and (b) pitch of 1.0. Each figure shows the conventional (top row) and motion corrected (middle row) scanning trajectories, using method of Sec. 2.5, in units of number of contributing views. The color bars indicate the range of values within each $3 \mathrm{D}$ volume, and the threshold set to $60 \%$ of the mean voxel value obtained with the conventional trajectory. Lower row: The corresponding motion corrected images $[\mathrm{WL}=-200 \mathrm{HU}, \mathrm{WW}=+2000 \mathrm{HU}]$. The prominent artifacts in the motion-corrected images with pitch of 1.0 correspond to regions of reduced sampling with the motion-corrected scanning trajectory.

interpolation between simulation and reconstruction, since the image was transformed during simulation, while the source/detector were transformed during reconstruction. The image quality at low pitch was superior, in particular in the presence of jitter. This may have been due to differences in axial sampling and object motion. Table 5 also revealed some degradation due to the jitter, and confirmed that lower pitch produced the most accurate motion corrected images.

\section{Discussion and Conclusions}

We have developed a method of retrospectively compensating for rigid motion in helical CT, and demonstrated its feasibility in simulations and CT scans acquired on a 16- 


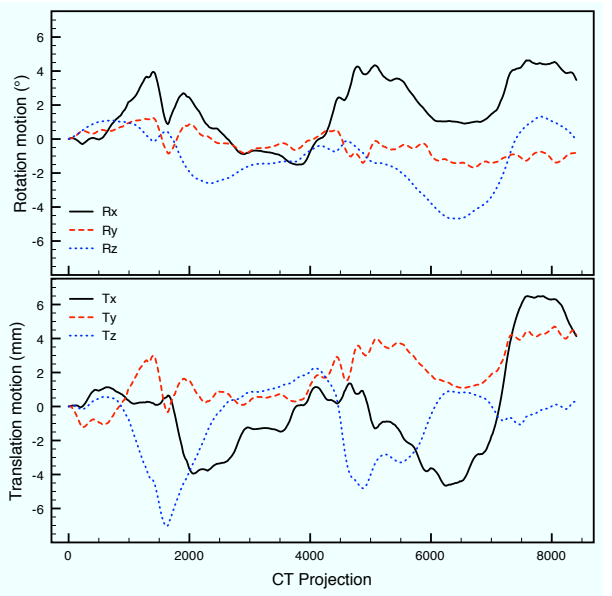

Figure 15. Rotations (top) and translations (bottom) of the 'true' volunteer head motion for $3 \mathrm{~s}($ pitch $=1.5)$.
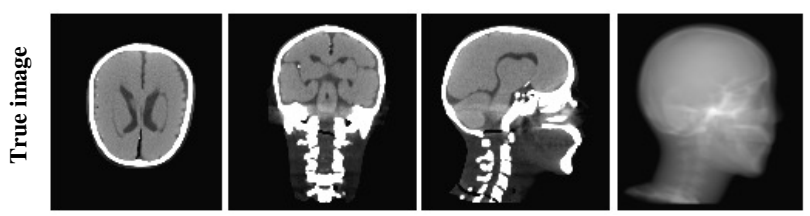

(a) Phantom

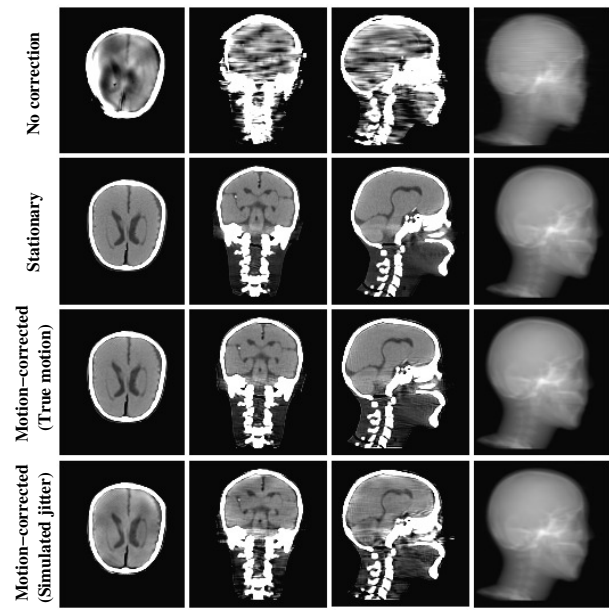

(b) Pitch $=0.5$

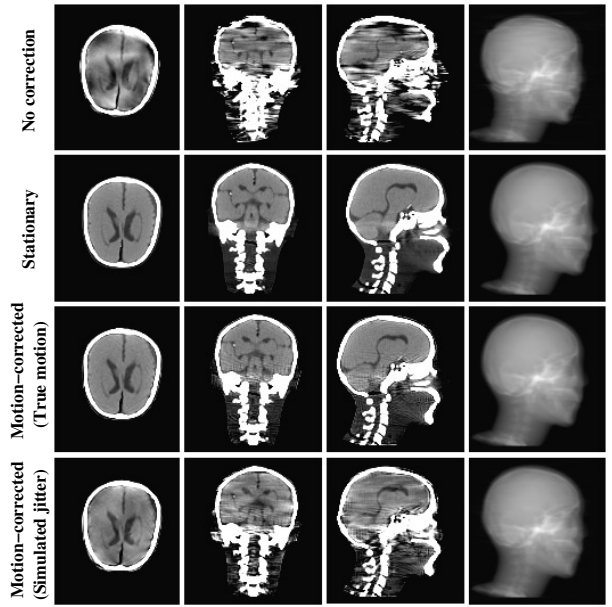

(c) Pitch $=1.5$

Figure 16. Motion correction in simulated CT studies with different pitch. (a) Voxelized phantom. (b) Pitch=0.5. (c) Pitch=1.5. In each panel the images in the right-hand column are projection images created by forward projection using a parallel beam projector. [ $W L=+40 \mathrm{HU}, W W=+140 \mathrm{HU}]$. 
Table 5. Motion correction accuracy in simulated CT scans with volunteer motion.

\begin{tabular}{lccc}
\hline Pitch 0.5 & $\overline{\mathrm{RMSE}}$ & $\overline{\mathrm{CC}}$ & $\overline{\mathrm{MSSIM}}$ \\
\hline No correction & $227.5(90.6)$ & $0.882(0.078)$ & $0.401(0.168)$ \\
Motion correction (no jitter) & $14.0(3.0)$ & $0.999(0.078)$ & $0.971(0.016)$ \\
Motion correction (jitter) & $39.4(10.1)$ & $0.997(0.001)$ & $0.862(0.042)$ \\
\hline Pitch 1.5 & $\overline{\mathrm{RMSE}}$ & $\overline{\mathrm{CC}}$ & $\overline{\mathrm{MSSIM}}$ \\
\hline No correction & $271.9(77.5)$ & $0.882(0.063)$ & $0.406(0.102)$ \\
Motion correction (no jitter) & $16.7(3.7)$ & $0.999(0.000)$ & $0.945(0.020)$ \\
Motion correction (jitter) & $71.7(22.4)$ & $0.991(0.006)$ & $0.769(0.050)$ \\
\hline
\end{tabular}

Values are mean (s.d.) of 95 transaxial slices.

slice clinical scanner. This method uses an optical motion tracking system to obtain six d.o.f. motion estimates during CT acquisition and reconstructs motion-corrected images using a fully 3D iterative MLTR reconstruction algorithm. The method restores projection consistency by spatially transforming the source and detector in response to rigid body motion and reconstructs the image from the resulting virtual scanner trajectory.

The most accurate motion corrected images in the real CT scans were obtained by acquiring motion data at the maximum tracker sampling rate, smoothing with an SG filter, linear interpolation, timestamp-based synchronization, and identifying and applying the optimal time shift. This yielded an appreciable improvement over the default motion correction method, reducing RMSE by $16 \%$ and increasing CC and MSSIM by $3 \%$ and $22 \%$, respectively. The residual RMSE in the fully optimised motion corrected image with pitch of 0.5 may be partly attributable to the heterogeneity of the materials comprising the phantom.

Proper synchronization of motion and CT data was essential for accurate motion correction. Assuming constant rates of acquisition of tracker data and CT projections did not appear to introduce serious errors. In the future, with the involvement of CT manufacturers, more precise synchronization could be achieved through hardware handshaking between the motion tracking system and CT scanner. Our method of ascertaining which tracker measurement corresponded to the start of the CT scan involved some uncertainty that resulted in a constant synchronization error that was eventually identifiable by trial and error. In the clinical situation, a motion-free scan will not be available, and an alternative method such as hardware handshaking will be needed for accurate synchronization.

There was a tradeoff between motion correction accuracy and reconstruction time with the ray-tracing and distance-driven (back)projectors. It has been shown that the distance-driven method generally provides better reconstruction quality (De Man and Basu, 2004). In cases without motion, the computation times of the ray-tracing and distance-driven (back)projector were comparable. However, motion destroyed the scanner symmetries, making our implementation of the distance-driven projector less efficient. Summing groups of four connective projections to reduce reconstruction 
time may have reduced the quality of our reconstructed images. For optimal results in clinical scans it would be better to accelerate the calculations using, for example, GPUs rather than reduce the sinogram size.

One limitation of the method is that motion correction can result in a scanning trajectory that is insufficient for exact reconstruction (Fulton et al., 1994, Clackdoyle, 2005). There was an indication of data insufficiency in a CT scan performed with a pitch of 1.0 in areas that corresponded to image artefacts. Whether this condition arises appears to depend on the object motion and the pitch, and when it did arise it affected some parts of the reconstructed volume more than others. In such cases the motion corrected images were still superior to the uncorrected ones, which indicates that even when exact reconstruction is not possible, a clinically useful image may still be obtainable in some cases.

No obvious data sufficiency artifacts were observed with volunteer head motion when a pitch of 1.5 was simulated, whereas they were seen in phantom scans at pitch of 1.0. This implies that the accuracy of the motion correction does not solely depend on pitch. Indeed it also depends on the nature of the object motion. The motion of the object was much larger in the phantom study performed with a pitch of 1.0, and data insufficiency may have been responsible for the appearance of artefacts in this case. This will be investigated in future work using additional measures of data insufficiency (Clackdoyle and Noo, 2001, Metzler et al., 2003, Liu et al., 2012). Furthermore, as we only studied one volunteer, data from many more volunteers is required to predict the likely incidence of data insufficiency in clinical use. If data sufficiency proves to be a problem in clinical scanning, it may be possible to explore solutions via techniques for reconstruction of incomplete projections such as projection-interpolation methods (Lewitt et al., 1978, Kalender et al., 1987, Srinivasa et al., 1997, Bang et al., 2011), the use of constrained reconstruction (Sidky et al., 2006), or projection correlation based view interpolation methods (Yan et al., 2010).

Ultimately an effective and practical motion correction method could streamline clinical workflows by eliminating the need for repeat scans in the event of motion. This would benefit patients prone to excessive motion who under current protocols are at risk of receiving an unnecessary radiation dose from repeat scans. The technique could benefit these patient groups not only in standalone CT, but also in hybrid imaging with PET/CT and SPECT/CT. Several groups, including our own, have implemented motion correction methods for neurological PET, using the Polaris motion tracking system, e.g. (Fulton et al., 2002, Bloomfield et al., 2003, Herzog et al., 2005). However, the possibility of also correcting for motion during the CT phase of hybrid imaging studies does not appear to have been envisaged previously. As we show here, the motion tracking equipment previously used in PET is also suited to CT scanning. Combining a CT motion correction method with existing PET motion correction methods, would enable motion correction to be applied to both the CT and PET phases of PET/CT. This is potentially quite significant: In PET/CT, motion during the CT scan affects not only the CT image but also the PET image, since accurate CT data are routinely relied upon for anatomical localization and attenuation correction of the emission data. The method could therefore potentially improve the accuracy of 
both the anatomical and functional images, even if head motion only occurs during the CT scan.

Our next goals are to evaluate this method in patient studies, and to further improve the method, for example by investigating methods of improving motion correction accuracy with incomplete projection data.

\section{Acknowledgments}

The authors would like to thank Dr. Karl Stierstorfer from Siemens AG, Healthcare Sector, Forchheim, Germany, for his valuable suggestions and help with the data format. We also thank Robbie Barnett, Scott Evans, David Skerrett and Krystal Moore of the Departments of Nuclear Medicine and Radiology, Westmead Hospital, for valuable technical and computing support. This work was supported by National Health and Medical Research Council grants 571378 and 632677, and by grant IWT 130065 of the Flemish agency for Innovation by Science and Technology. J. Kim is supported by a University Postgraduate Award from the University of Sydney.

\section{References}

Al-Shakhrah I and Al-Obaidi T 2003 Common artifacts in computerized tomography: A review Appl. Radiol. 32(8) 25-30

Bang T and Jeon I 2011 CT reconstruction from a limited number of X-ray projections WASET 58 627-9

Beyer T, Tellmann L, Nickel I and Pietrzyk U 2005 On the use of positioning aids to reduce misregistration in the head and neck in whole-body PET/CT studies $J$. Nucl. Med 46(4) 596-602

Bhowmik U K, Iqbal M Z and Adhami R R 2012 Mitigating motion artifacts in FDK based 3D cone-beam brain imaging system using markers Cent. Eur. J. Engl. 2(3) 369-92

Bloomfield P, Spinks T, Reed J, Schnorr L, Westrip A, Livieratos L, Fulton R and Jones T 2003 The design and implementation of a motion correction scheme for neurological PET Phys. Med. Biol. 48(8) 959-78

Bodensteiner C, Darolti C, Schumacher H, Matthäus L and Schweikard A 2007 Motion and positional error correction for cone beam 3D-reconstruction with mobile C-arms 10th International Conference on Medical Image Computing and Computer Assisted Intervention - MICCAI 2007, Brisbane, Australia

Brenner D J, Elliston C D, Hall E J and Berdon W E 2001 Estimated risk of radiationinduced fatal cancer from pediatric CT Am. J. Roentgenol 176(2) 289-96

Brenner D J and Hall E J 2007 Computed tomography - an increasing source of radiation exposure N. Engl. J. Med 357 2277-84

Brenner D J 2010 Slowing the Increase in the Population Dose Resulting from CT Scans Radiat. Res. 174(6b) 809-15 
Clackdoyle R and Noo F 2001 Cone-beam tomography from 12 pinhole vertices 2001 IEEE Nuclear Science Symposium Conference Record, 4 1874-76

Clackdoyle R 2005 Fully 3D reconstruction theory in perspective Proceedings of the Eighth International Meeting on Fully Three-Dimensional Image Reconstruction in Radiology and Nuclear Medicine, Salt Lake City, Utah, USA, 64-69

Crawford C, King K, Ritchie C and Godwin J 1996 Respiratory compensation in projection imaging using a magnification and displacement model IEEE Trans. Med. Imag. 15(3) 327-32

De Man B and Basu S 2004 Distance-driven projection and backprojection in three dimensions Phys. Med. Biol. 49(11) 2463-75

Desbat L, Rit S, Clackdoyle R, Mennessier C, Promayon E and Ntalampeki S 2007 Algebraic and analytic reconstruction methods for dynamic tomography 2007 IEEE Nuclear Science Symposium Conference Record 726-30

Desjardins B and Kazerooni E A 2004 ECG-gated cardiac CT Am. J. Roentgen. 182(4) 993-1010

Edlow J A and Caplan L R 2000 Avoiding pitfalls in the diagnosis of subarachnoid hemorrhage N. Engl. J. Med. 342(1) 29-36

Elfring R, de la Fuente M and Radermacher K 2010 Assessment of optical localizer accuracy for computer aided surgery systems Comp. Aid. Surg. 15(1-3) 1-12

Ens S, Ulrici E, Hell J and Buzug T 2010 Automatic motion correction in cone-beam computed tomography 2010 IEEE Nuclear Science Symposium Conference Record $3248-51$

Fahmi F, Beenen L F M, Streekstra G J, Janssen N Y, de Jong H W, Riorda A, Roos Y B, Majoie C B, Van Bavel E and Marquering H A 2013 Head movement during $\mathrm{CT}$ brain perfusion acquisition of patients with suspected acute ischemic stroke Eur. J. Radiol. 82 2334-41

Fulton R R, Hutton B F, Braun M, Ardekani B and Larkin R 1994 Use of 3D reconstruction to correct for patient motion in SPECT Phys. Med. Biol. 39 563-74

Fulton R, Meikle S, Eberl S, Pfeiffer J, Constable C and Fulham M 2002 Correction for head movements in positron emission tomography using an optical motion-tracking system IEEE Trans. Nucl. Sci. 49(1) 116-23

Herzog H, Tellmann L, Fulton R, Stangier I, Rota-Kops E, Bente K, Boy C, Hurlemann R and Pietrzyk U 2005 Motion artifact reduction on parametric PET images of neuroreceptor binding J. Nucl. Med. 46(6) 1059-65

Hoffman E J, Cutler P D, Digby W M and Mazziotta J C 1990 3D phantom to simulate cerebral blood flow and metabolic images for PET IEEE Trans. Nucl. Sci. 37(2) 616-20

Isola A A, Ziegler A, Koehler T, Niessen W J and Grass M 2008 Motion-compensated iterative cone-beam CT image reconstruction with adapted blobs as basis functions Phys. Med. Biol. 53(23) 6777-97 
Jacobson M W and Stayman J W 2008 Compensating for head motion in slowlyrotating cone beam CT systems with optimization transfer based motion estimation 2008 IEEE Nuclear Science Symposium Conference Record 5240-5

Joseph P M 1982 An improved algorithm for reprojection rays through pixel images IEEE Trans. Med. Imag. MI-1(3) 193-6

Kalender W A, Hebel R and Ebersberger J 1987 Reduction of CT artifacts caused by metallic implants Radiol. $164576-7$

Kaste S C 2004 Issues specific to implementing PET/CT for pediatric oncology: what we have learned along the way Pediatr. Radiol. 34(3) 205-13

Katsuda T, Eiho S, Kuroda C and Hashimoto T 2005 Analysis of breath holding for lung CT imaging Radiography 11(4) 235-41

Kim J-H, Nuyts J, Kuncic Z and Fulton R 2013 The feasibility of head motion tracking in helical CT: A step toward motion correction Med. Phys. 40(4) 041903-1-4

Kyme A Z, Zhou V W, Meikle S R and Fulton R R 2008 Real-time 3D motion tracking for small animal brain PET Phys. Med. Biol. 53(10) 2651-66

Kyme A Z, Zhou V W, Meikle S R, Baldock C and Fulton R 2011a Optimised motion tracking for positron emission tomography studies of brain function in awake rats PLoS ONE 6(7) e21727

Lee B and Newberg A 2005 Neuroimaging in traumatic brain imaging NeuroRX 2(2) $372-83$

Lewitt R M and Bates R H T 1978 Image reconstruction from projections iii: Projection completion methods (theory) Optik 50 189-204

Linney N C and Gregson P H 2001 Organ motion detection in CT images using opposite rays in fan-beam projection systems IEEE Trans. Med. Imag. 20(11) 110922

Liu B, Bennett J, Wang G, De Man B, Zeng K, Yin Z, Fitzgerald P, and Yu H 2012 Completeness map evaluation demonstrated with candidate next-generation cardiac CT architectures Med. Phys. 39(5) 2405-16

Lu W and Mackie T 2002 Tomographic motion detection and correction directly in sinogram space Phys. Med. Biol. 47 1267-84

Malviya S, Voepel-Lewis T, Eldevik OP, Rockwell DT, Wong JH and Tait AR 2000 Sedation and general anaesthesia in children undergoing MRI and CT: adverse events and outcomes Br. J. Anaesth. 84(6) 743-8

Marchant T E, Price G J, Matuszewski B J and Moore C J 2011 Reduction of motion artefacts in on-board cone beam CT by warping of projection images $\mathrm{Br}$. J. Radiol. 84(999) 251-64

Mennessier C, Clackdoyle R and Noo F 2009 Direct determination of geometric alignment parameters for cone-beam scanners Phys. Med. Biol. 54(6) 1633-60

Metzler S, Greer K and Jaszczak R 2003 Helical pinhole SPECT for small-animal imaging: A method for addressing sampling completeness IEEE Trans. Nucl. Sci. 50(5) $1575-83$ 
Noo F, Clackdoyle R, Mennessier C, White T A and Roney T J 2001 Analytic method based on identification of ellipse parameters for scanner calibration in cone-beam tomography Phys. Med. Biol. 45(11) 3489-3508

Nuyts J, Man D B, Dupont P, Defrise M, Suetens P and Mortelmans L 1998 Iterative reconstruction for helical CT: A simulation study Phys. Med. Biol. 43 729-37

Paul R 1982 Robot Manipulators: Mathematics, Programming and Control MIT Press, Cambridge, MA

Picard Y and Thompson C 1997 Motion correction of PET images using multiple acquisition frames IEEE Trans. Med. Imag. 16(2) 137-44

Popilock R, Sandrasagaren K, Harris L and Kaser K A 2008 CT Artifact Recognition for the Nuclear Technologist J. Nucl. Med. Technol. 36(2) 79-81

Rit S, Sarrut D and Desbat L 2009 Comparison of analytic and algebraic methods for motion-compensated cone-beam CT reconstruction of the thorax IEEE Trans. Med. Imag. 28(10) 1513-25

Ritchie C, Hsieh J and Gard M 1994 Predictive respiratory gating: A new method to reduce motion artifacts on CT scans Radiol. 190 847-52

Ritchie C, Crawford C, Gowin J, King K and Kim Y 1996 Correction of computed tomography motion artefacts using pixel-specific back projection IEEE Trans. Med. Imag. 15(3) 333-42

Roux S, Desbat L, Koenig A and Grangeat P 2004 Exact reconstruction in 2-D dynamic CT: Compensation of time-dependent affine deformations Phys. Med. Biol. 49(11) 2169-82

Savitzky A and Golay M J E 1964 Smoothing and differentiation of data by simplified least squares procedures Anal. Chem 36(8) 1627-39

Schäfer D, Bertram M, Conrads N, Wiegert J, Rose G and Rasche V 2004 Motion compensation for cone-beam $\mathrm{CT}$ based on $4 \mathrm{D}$ motion field of sinogram tracked markers Int. Congr. Ser. 1268 189-94

Schöndube H, Allmendinger T, Stierstorfer K, Bruder H and Flohr T 2013 Temporal resolution and motion artifacts in single-source and dual-source cardiac CT Med. Phys. 40(3) 031112

Schretter C, Rose G and Bertram M 2009 Image-based iterative compensation of motion artifacts in computed tomography Med. Phys. 36(11) 5323

Sidky E Y, Kao C M and Pan X H 2006 Accurate image reconstruction from few-views and limited-angle data in divergent-beam CT J. X-Ray Sci. Technol. 14 119-39

Srinivasa N, Ramakrishnan K and Rajgopal K 1997 Image reconstruction from incomplete projection J. Med. Life Sci. Eng. 14 1-19

Stavdahl O, Bondhus A K, Pettersen K Y and Malvig K E 2005 Optimal statistical operators for 3-dimensional rotational data: geometric interpretations and application to prosthesis kinematics Robotica 23(3) 283-92

Taguchi K and Kudo H 2008 Motion compensated fan-beam reconstruction for nonrigid transformation IEEE Trans. Med. Imag. 27(7) 907-17 
Van Slambrouck K and Nuyts J 2012 Metal artifact reduction in computed tomography using local models in an image block-iterative scheme Med. Phys. 39(11) 7080-93

Vassileva J, Rehani M M, Al-Dhuhli $\mathrm{H}$ and et al. 2012 IAEA Survey of Pediatric CT Practice in 40 Countries in Asia, Europe, Latin America, and Africa: Part 1, Frequency and Appropriateness Am. J. Roentgenol. 198(5) 1021-31

Wachtel R E, Dexter F and Dow A J 2009 Growth rates in pediatric diagnostic imaging and sedation Anesth. Analg. 108(5) 1616-21

Wagner A, Schicho K, Kainberger F, Birkfellner W, Grampp S and Ewers R 2003 Quantification and clinical relevance of head motion during computed tomography Invest. Radiol. 38 733-41

Wang G and Vannier M W 1995 Preliminary study on helical CT algorithms for patient motion estimation and compensation IEEE Trans. Med. Imag. 14(2) 205-11

Wang Z, Bovik A, Sheikh H and Simoncelli E 2004 Image quality assessment: From error visibility to structural similarity IEEE Trans. Imag. Process 13(4) 600-12

Weisenberger A G, Gleason S S, Goddard J, Kross B, Majewski S, Meikle S R, Paulus M J, Pomper M, Popov V, Smith M F, Welch B L and Wojcik R 2005 A restraint-free small animal SPECT imaging system with motion tracking IEEE Trans. Nucl. Sci. 52(3) 638-44

Westermann B and Hauser R 2000 Online head motion tracking applied to the patient registration problem Comp. Aid. Surg. 5(3) 137-47

Yan H, Mou X, Tang S, Xu Q and Zankl M 2010 Projection correlation based view interpolation for cone beam CT: primary fluence restoration in scatter measurement with a moving beam stop array Phys. Med. Biol. 55 6353-6375

Yazdi M and Beaulieu L 2008 Artifacts in spiral x-ray CT scanners: Problems and solutions Int. J. Biol. Life Sci. 4(3) 135-39

$\mathrm{Yu} \mathrm{H}$ and Wang G 2007 Data consistency based rigid motion artifact reduction in fan-beam CT IEEE Trans. Med. Imag. 26 249-60

Zafar S 2011 Post scan correction of step, linear and spiral motion effects in CT scans Int. J. Comput. Appl. 35(10) 13-19 